\section{Nanoseconds molecular dynamics simulation of primary mechanical energy transfer steps in $F_{1}$-ATP synthase}

Rainer A. Böckmann and Helmut Grubmüller

Theoretical Molecular Biophysics Group, Max-Planck-Institute for Biophysical Chemistry, Am Fassberg 11, 37077 Göttingen, Germany.

Published online: 11 February 2002, DOI: 10.1038/nsb760

The mitochondrial membrane protein $\mathrm{F}_{0} \mathrm{~F}_{1}$-ATP synthase synthesizes adenosine triphosphate (ATP), the universal currency of energy in the cell. This process involves mechanochemical energy transfer from a rotating asymmetric $\gamma$-'stalk' to the three active sites of the $F_{1}$ unit, which drives the bound ATP out of the binding pocket. Here, the primary structural changes associated with this energy transfer in $F_{1}-A T P$ synthase were studied with multi-nanosecond molecular dynamics simulations. By forced rotation of the $\gamma$-stalk that mimics the effect of proton motive $F_{0}$-rotation during ATP synthesis, a time-resolved atomic model for the structural changes in the $F_{1}$ part in terms of propagating conformational motions is obtained. For these, different time scales are found, which allows the separation of nanosecond from microsecond conformational motions. In the simulations, rotation of the $\gamma$-stalk lowers the ATP affinity of the $\beta_{\mathrm{TP}}$ binding pocket and

Fig. 1 ATP synthase structure, function and simulation setup. a, A slice of $F_{1}$ at the level of the active sites, viewed from the cytosolic side. $\boldsymbol{b}, \mathrm{F}_{\mathrm{o}} \mathrm{F}_{1}-\mathrm{ATP}$ synthase with the atomically resolved $\mathrm{F}_{1}$ simulation system. $A$ proton flux through the membrane-bound $F_{0}$ unit (red) drives rotation of the asymmetric $\gamma$-stalk (orange), with respect to the $F_{1}$ unit (green and cyan) located in the mitochondrial matrix. Co-rotation of $F_{1}$ is hindered by $\delta$ and $b$ (yellow), the exact positioning of which is not generally agreed upon. This rotation triggers conformational changes mainly within the three $\beta$-subunits, DP, TP and E (green), which bind ADP and phosphate and subsequently drive ATP synthesis and release. These changes concern mostly the orientation of the lower-half of the $\beta$-subunit (light green), which can tilt with respect to the upper-half (dark green). Accordingly, the conformation seen for the empty subunit, $\beta_{E}$, is denoted 'open', whereas $\beta_{T P}$ and $\beta_{\mathrm{DP}}$ are referred to as 'closed' 6 . After $120^{\circ}$ rotation of $\gamma$, DP, TP and E switch roles clockwise. Our molecular dynamics simulations included the $F_{1}$ unit $(\alpha \beta)_{3} \gamma$ (except $\delta$ and b), bound nucleotides as shown in panel (a), a large solvation shell (blue) and salt ions (red and yellow), comprising a total of 183,674 atoms. C, In the simulations, torque was exerted onto the lower part of $\gamma$ by subjecting the respective backbone atoms (filled circle) to restraining potentials (open circle), which are moved with constant angular velocity $\omega$ along concentric circles around the pseudo symmetry axis (circle with ' $x$ ') of the complex. Throughout the simulations, co-rotation of the $(\alpha \beta)_{3}$ complex was prevented by immobilizing the centers of mass of the putative $\delta$ contact region of two $\alpha$-subunit head groups (residues $\alpha_{T P}$ 24-130 and $\alpha_{D P}$ 19-96), thereby mimicking the effect of the $\delta$ 'stator'. To prevent tilt of the whole system, the center of mass of residues $\alpha_{E} 255-265$ and 310-320 were immobilized. $\boldsymbol{d}$, The torque required to move the individual residues of $\gamma$ within $(\alpha \beta)_{3}$ (black = zero or negative torque and yellow $=$ $0.7 \times 10^{-18} \mathrm{Nm}$ ) allows the identification of hot spots of mechanical energy transfer. Mainly due to Stokes' friction, the averaged torque is larger by a factor of $\sim 50$ than the measured average torque of $5 \times 10^{-20} \mathrm{Nm}$ (ref. 22). Comparison of that value with the value of $1.02 \times 10^{-18} \mathrm{Nm}$ obtained from an additional simulation with four-fold lower angular velocity $\left(\omega=29^{\circ} \mathrm{ns}^{-1}\right)$ yields a velocity-dependent frictional torque contribution of $(2.0 \pm 0.3) \times 10^{-18} \mathrm{Nm}$. The associated dissipated energy raises the temperature of the $\gamma$-subunit by $<1 \mathrm{~K}$. triggers fast, spontaneous closure of the empty $\beta_{\mathrm{E}}$ subunit. The simulations explain several mutation studies and the reduced hydrolysis rate of $\boldsymbol{\gamma}$-depleted $F_{1}$-ATPase.

$\mathrm{F}_{\mathrm{o}} \mathrm{F}_{1}$-ATP synthase (Fig. 1 $1 a, b$ ) utilizes the electrochemical potential of a transmembrane proton gradient to synthesize $\mathrm{ATP}^{1,2}$. Notably, energy conversion involves mechanochemical energy transfer from a rotating asymmetric $\gamma$-'stalk' over a distance of $>2.5 \mathrm{~nm}$ to the three ATP synthesis sites of the $\mathrm{F}_{1}$ complex (Fig. $1 a)^{3-5}$. X-ray structures of the $\mathrm{F}_{1}$ unit $^{6,7}$ show its three catalytic $\beta$-subunits in different conformational states, TP, DP and E, with bound ATP (analog), ADP and empty binding site, respectively ${ }^{6}$. The structures suggest that the energy consuming step in the ATP synthesis cycle - that is, ATP release - is driven by concerted conformational motions within the $(\alpha \beta)_{3}$ complex, which are dictated by the changing orientation of the asymmetric $\gamma$ subunit inside $(\alpha \beta)_{3}$ (ref. 6), thus rendering $\mathrm{F}_{0} \mathrm{~F}_{1}$-ATP synthase a spectacular example of a 'molecular machine'.

The central metabolic role and high efficiency of this machine has triggered much experimental work on substrate-binding affinities $^{8}$, thermodynamics, stoichiometry ${ }^{9}$ and kinetics ${ }^{8}$, as well as on mutants $\mathrm{s}^{8,10,11}$ and crosslinks ${ }^{3}$. To combine these data into coherent pictures, several simplified models without incorporating atomic detail have been discussed ${ }^{10,12-16}$. Here we study at the atomic level how these motions are induced by rotation of the $\gamma$-stalk, how and along which route they propagate towards the nucleotide binding sites, and why and in what sequence the observed structural changes lower ATP binding affinity at $\beta_{\mathrm{TP}}$, as is required for ATP to leave the binding pocket after synthesis. Aiming at model-independent answers to these questions, we have performed molecular dynamics force probe simulations ${ }^{17,18}$ of the fully solvated $(\alpha \beta)_{3} \gamma$ complex (Fig. $1 b$ ), which is the minimal complex capable of normal ATPase activity ${ }^{19,20}$. In the simulations, the lower part of the $\gamma$-stalk was forced to rotate (Fig. 1c)

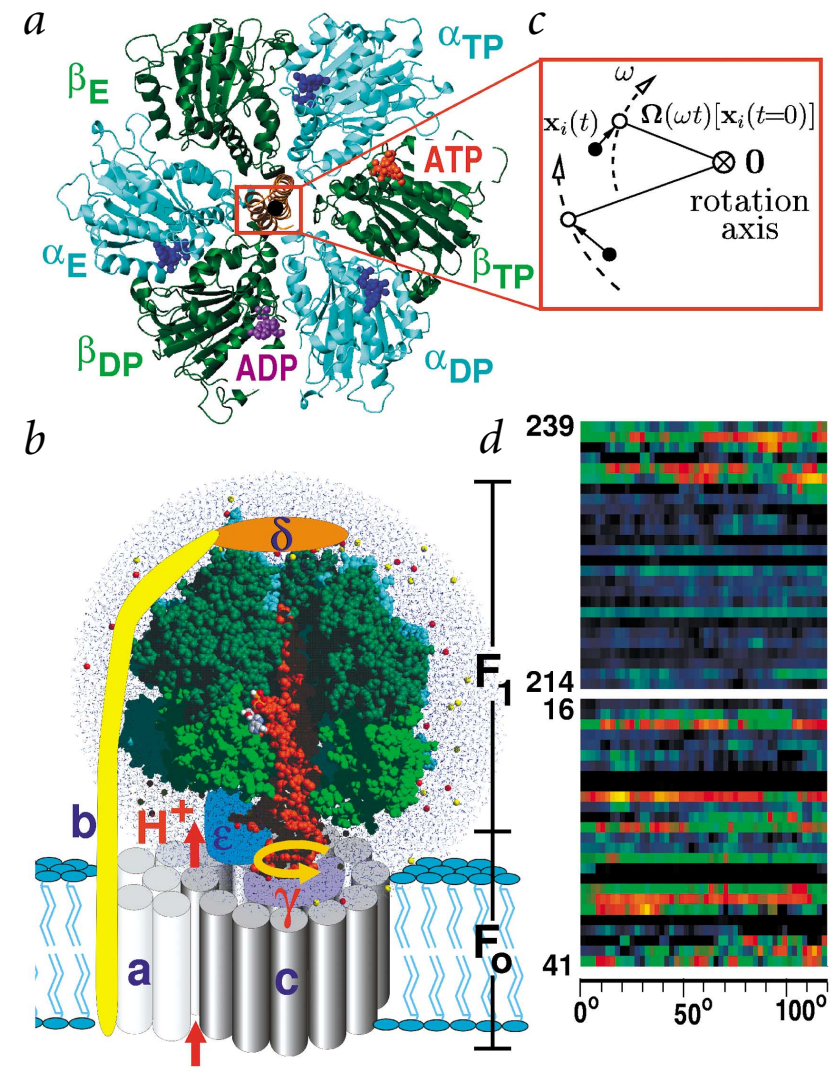


$a$

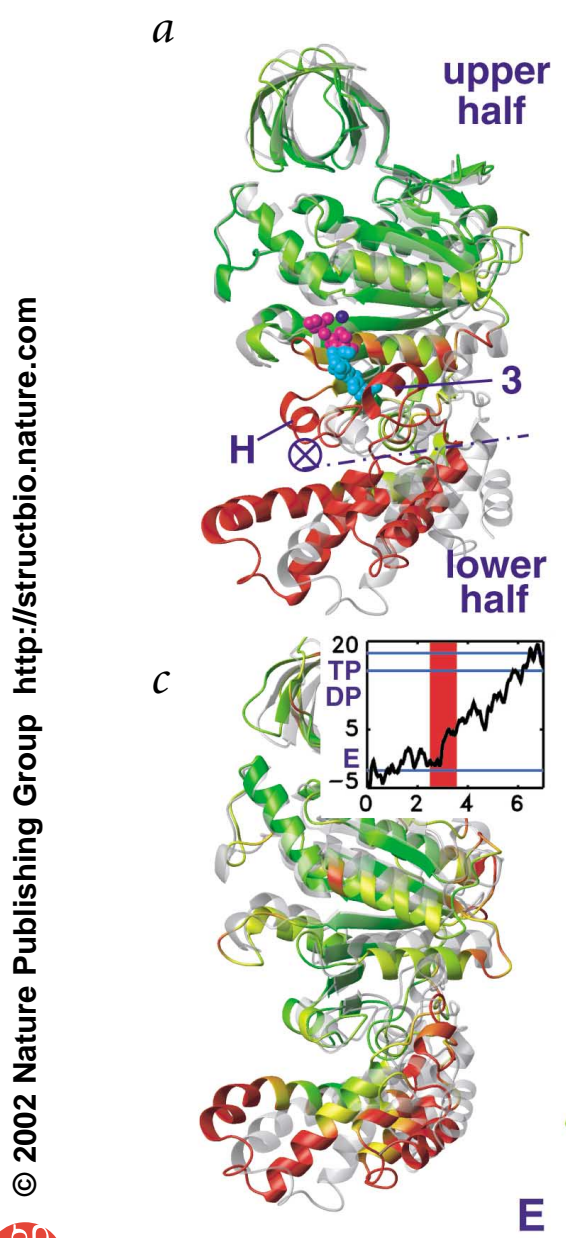

$b$
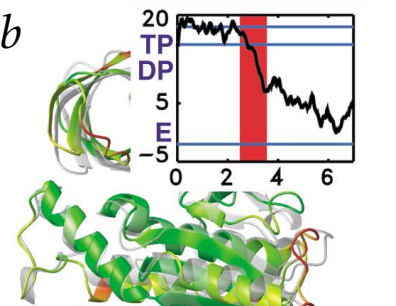

to mimic the $\mathrm{F}_{\mathrm{o}}$ rotation caused in vivo by the proton motive force; the results reveal the subsequent conformational motions triggered from this rotation.

Due to the huge computational cost of such simulations, the time span covered is limited to several nanoseconds - that is, considerably shorter than the microseconds time scale at which these conformational motions presumably take place in vivo ${ }^{21}$. Accordingly, torques larger than the measured ones ${ }^{22}$ by factors of 12 and 50 had to be applied in the simulations. Furthermore, slow (microsecond) conformational motions are unlikely to show up. For the faster motions that are seen in the simulations, however, the large amount of available experimental data allowed us to verify that these motions are described correctly.

\section{Forced $120^{\circ}$ rotation of the $\gamma$-subunit}

During an initial equilibration phase, the system remained close to the X-ray structure. In particular, the hydrogen bonding networks between the nucleotides and the residues of the respective binding pockets remained intact, and the $\mathrm{Mg}^{2+}$ coordination, as well as ligated water molecules, displayed the main features of the X-ray model ${ }^{23}$. Furthermore, the fluctuations of the individual binding pocket residues during equilibration agree well with those obtained from the averaged temperature factors eight other X-ray structures of ATPase (PDB entries 1BMF, 1E79, 1COW, 1E1Q, 1E1R, 1EFR, 1H8H and 1NBM), with correlation coefficients of $0.84,0.68$ and 0.70 for the empty, ATP and ADP pocket, respectively.

After equilibration, a $120^{\circ}$ force probe rotation simulation in the synthesis direction was carried out with an angular velocity

Fig. 2 Structural changes of the $\beta$-subunits induced by enforced rotation of the $\gamma$-stalk. The size of these changes is color-coded (green $=$ small and red $=$ large). a, Overlay of $\beta_{\text {TP }}$ (colored) and $\beta_{\mathrm{E}}$-subunits (gray,) taken from the $\mathrm{X}$-ray structure, in closed and open conformation, respectively (rotation axis $=$ circle with ' $\times$ '). $\boldsymbol{b}-\boldsymbol{d}$, Changes in $\beta_{T P}, \beta_{E}$ and $\beta_{D P}$, respectively, as induced by enforced $120^{\circ}$ rotation and subsequent free dynamics. Shown are the initial (X-ray) structures (gray) and final structures (colored). The insets show the rotation angle between upper and lower part of the respective $\beta$-subunits in the course of the $7 \mathrm{~ns}$ simulation. The red bar indicates the period of enforced rotation, and green lines denote the three $\beta$-subunit angles of the equilibrated $X$-ray structure.

of $\omega=115^{\circ} \mathrm{ns}^{-1}$ (Fig. 1 c). During a subsequent relaxation period of $3.5 \mathrm{~ns}$, within which the lower part of the $\gamma$-subunit was kept restrained to avoid back-rotation, the induced conformational motions propagated further towards the active sites. The torques exerted onto the individual $\gamma$-residues during the $120^{\circ}$ forced rotation (Fig. 1d) show peaks, reflecting energy transfer to $(\alpha \beta)_{3}$, dissipation and enforced crossing of associated energy barriers. This crossing is largely nonactivated at the nanosecond simulation time scale; therefore, the peaks should be more pronounced in our torque profiles than they would appear at physiological turnover rates.

Most of the C-terminal $\gamma$-helix (residues 214-233) rotates almost freely. Residues 234-238, which form the middle part of the $\gamma$-stalk and are located close to the DELSEED region of the $\beta_{\mathrm{TP}}$ subunit, exhibit larger torques mainly due to steric interactions, which bend away the helix-coil(DELSEED)-helix region of $\beta_{\mathrm{TP}}$. For the reverse ATPase direction, this implies considerable energy transfer from the ATP-loaded $\beta$-subunit to $\gamma$, in agreement with accepted models ${ }^{10,12-16}$. For the N-terminal $\gamma$-helix, the apparent torque peak at $30^{\circ}$ for Arg 36 is caused by the rupture of a hydrogen bonding network between $\gamma$ (residues Lys 30, Arg 33 and Arg 36) and Asp 409 from $\alpha_{E}$, as well as the $\beta_{\mathrm{E}}$-DELSEED region. Here the entire load apparently rests on the lowermost $\gamma$-residues, Arg 36 and Glu 35, due to the intrinsic elasticity of the $\gamma$-helix. Steric interactions also occur, mainly between Met 25 and the $\gamma$-proximal part of helix 1 (Fig. 4), giving rise to the second pronounced peak. For Lys 18, water bridges cause moderate forces. The breaking of hydrogen bonds between $\gamma(\operatorname{Arg} 228)$ and $\beta_{\mathrm{DP}}$ (Glu 395) at $50^{\circ}$ is not seen in the torque profiles.

\section{Opening and closing motions}

Major changes are seen in two of the three $\beta$-subunits during enforced rotation and subsequent free dynamics (Fig. 2). The lower part of $\beta_{\mathrm{TP}}$ is pushed sideways toward $\alpha_{\mathrm{DP}}$ and downward toward an open configuration (Fig. 2b). This observation is quantified by the change of the bending angle (Fig. 2, inset) between the two domains separated by the dashed line in Fig. $2 a$, which approaches that seen in $\beta_{\mathrm{E}}$. Simultaneously, an upwards rotation of the lower half of $\beta_{\mathrm{E}}$ towards the closed X-ray conformation of $\beta_{\mathrm{TP}}$ and $\beta_{\mathrm{DP}}$ is observed (Fig. $2 c$ ). Initially, this motion is sterically obstructed by both the $\gamma$-subunit and $\beta_{\mathrm{TP}}$ and, therefore, proceeds synchronously to the removal of these obstacles. Although this motion proceeds very fast, it is apparently not driven by the $\gamma$-rotation, in contrast to the motion in $\beta_{\mathrm{TP}}$. In this sense, this spontaneous motion resembles the relaxation of a spring. In particular, substrate binding is not required. In confirmation, $\beta_{\mathrm{TP}}$ (including bound substrate) remained closed in additional simulations of isolated solvated $\beta$-subunits, whereas $\beta_{\mathrm{E}}$ closed spontaneously within $3 \mathrm{~ns}$ (data not shown). These findings imply that, for the reverse hydrolysis cycle, opening of $\beta_{\mathrm{E}}$ is an energy-consuming step that is driven, via $\gamma$-rotation, by 
Fig. 3 Changes at the catalytic $\alpha-\beta$ interface. Helix 3 (residues $418-426$ ) of each $\beta$-subunit is highlighted in blue. $\boldsymbol{a}$, Open empty and $\boldsymbol{b}$, closed TP conformation, as seen in the X-ray structure that has been used as starting structure for the simulations. $c, \alpha_{E}-\beta_{E}$ interface from the simulation after enforced $\gamma$-rotation and equilibration. $\boldsymbol{d}$, A recent half-closed structure with bound ADP and sulfate, which has been proposed as an intermediate 24 .

conformational motions of one or both of the other $\beta$-subunits — either by ATP binding or by phosphate release ${ }^{16}$. Without $\gamma$-coupling, $\beta_{\mathrm{E}}$ would likely get trapped in closed conformation. Indeed, the hydrolysis rate of $\gamma$-depleted $(\alpha \beta)_{3}$ is drastically reduced ${ }^{20}$.

Significantly smaller changes are seen for $\beta_{\mathrm{DP}}$ (Fig. $2 d$ ), where the orientation of the lower part is nearly unchanged. Exceptions to this are helices 5 and 6 , which bend slightly outwards, as well as the $\gamma$-proximal part of helix G. For all three $\beta$-subunits, considerable fluctuations are seen for the short helix 3 at the adenine pocket, residues $418-426$, in agreement with the disorder seen for these residues in the $\beta_{\mathrm{E}} \mathrm{X}$-ray structure. Overall, the opening and closing motions of the lower halves of the $\beta$-subunits are nearly completed within the 7 ns simulation time span, without being subjected to any restraining potential. Apparently, the frictional forces are weak enough to allow even the observed large motions of the lower $\beta$-halves within nanoseconds.

Despite the spontaneous closure of the $\beta_{\mathrm{E}}$ subunit, the $\alpha_{\mathrm{E}}-\beta_{\mathrm{E}}$ catalytic interface remains open. This can be seen by comparing the positions of helix 3 (Fig. 3, blue) for the open $\alpha_{\mathrm{E}}-\beta_{\mathrm{E}}$ (Fig. 3a) and the closed $\alpha_{\mathrm{TP}}-\beta_{\mathrm{TP}}$ (Fig. $3 b$ ) catalytic interfaces from the crystal structure, with the respective position seen after enforced rotation for $\beta_{\mathrm{E}}$ (Fig. $3 c$ ). Comparing the structure with the recently solved half-closed $\beta_{\mathrm{ADP}+\mathrm{Pi}}$ conformation ${ }^{24}$ (Fig. $3 d$ ) is also instructive. Here, the catalytic interface is closed even though the $\beta$-subunit adopts an open conformation similar to the $\beta_{\mathrm{E}}$ subunit (Fig $3 a$ ). Comparison of the X-ray structures of $\beta_{\mathrm{E}}$ and $\beta_{\mathrm{ADP}+\mathrm{Pi}}$ reveals a rotation of the $\mathrm{C}$-terminal domain around the axis of the $(\alpha \beta)_{3}$ subcomplex, approximately perpendicular to the rotation axis that connects the open with the closed conformation ${ }^{24}$. A similar 'sideways bending', in reverse direction, is seen in our simulation for the lower half of the $\beta_{\mathrm{TP}}$ subunit, which is driven here by the forced $\gamma$-rotation in the synthesis direction. In contrast to the half-closed crystal structure, which was interpreted as a hydrolysis intermediate before ADP release $^{24}$, the spontaneously closed $\beta_{\mathrm{E}}$ subunit probably resembles a synthesis intermediate before ADP binding.

\section{Triggered sequence of conformational changes}

Next, we analyzed the details and timing of the conformational motions in $\beta_{\mathrm{TP}}$ (Fig. 4), which are triggered by $\gamma$-subunit rotation. Overall, a domino-like propagation of structural changes towards the ATP-binding site is observed, which proceeds much slower than an elastic wave and just below the velocity at which frictional forces in proteins are found to become dominant ${ }^{17,18,25}$. Accordingly, friction should be negligible at physiological time scales, in agreement with the $\sim 100 \%$ efficiency of the enzyme ${ }^{14}$.

Closer inspection reveals that the structural changes propagate along two main routes toward the ATP-binding site and, therefore, are expected to affect the energy transfer required for the decrease in ATP binding affinity. The dominant route (red, Fig. 4) leads via the DELSEED region and the adjacent helices 1 and 2, as well as via the $\beta_{\mathrm{TP}}$ hinge region (residues 136-144 and 354-359), toward the binding pocket. Here, three steps can be distinguished. Almost synchronously with the $\gamma$-rotation, the $\gamma$-proximal part of helix 1 and, subsequently helix 2 , are pushed
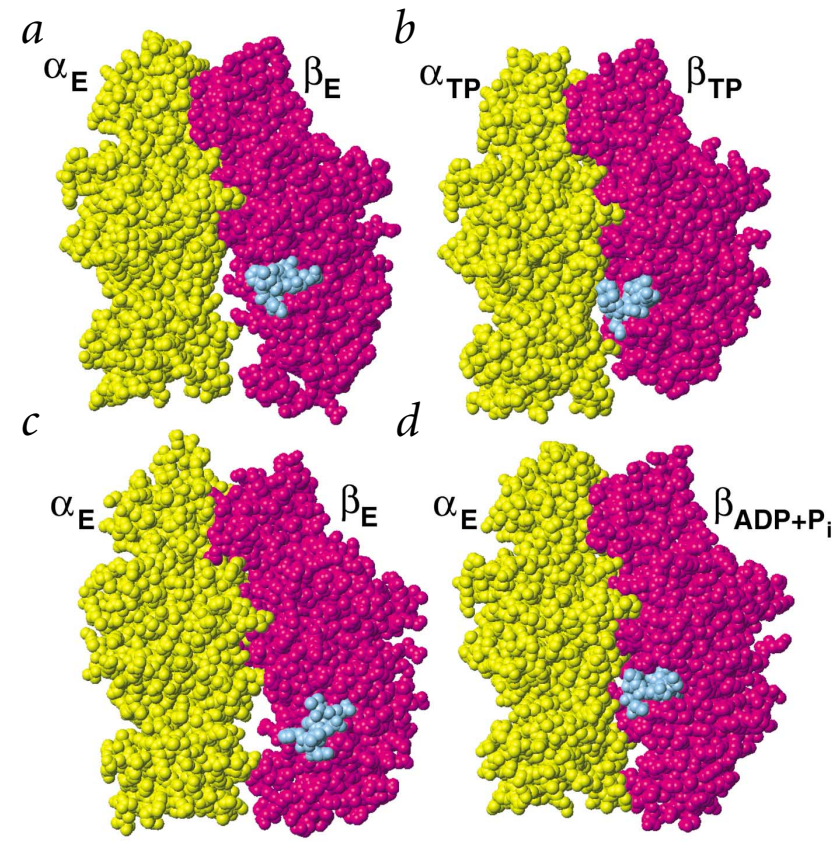

downward and sideways. This motion is dominated by steric interactions, which is consistent with the observation that the generated torque is nearly unaffected in mutants with decreased negative charge in the highly charged DELSEED region ${ }^{11}$. These motions drive a second step, during which the more distal parts of helices 1 and 2 start to move. At this time, the hinge region undergoes complex structural changes. In a third step, these changes drive helix $\mathrm{B}$, which is located at the binding site, outward as it becomes more tilted with respect to helix $\mathrm{C}$. The second, less pronounced route of structural changes (green, Fig. 4) involves the hydrophobic bearing (residues 273-281) of $\gamma$ (region E) and region G (Fig. 4). These are pushed sideways by $\gamma$, such that hydrogen bonds between $\beta_{\mathrm{TP}}$ Asp 315 and Asp 316 to $\gamma$ Arg 252 are formed. Subsequently, helix $\mathrm{H}$ is pushed slightly downward, and the two strands of $\beta$-sheet 7 start to disrupt. Presumably, these motions also contribute to shifting helix B outward.

As with the overall tilting motion of the lower half of $\beta_{\mathrm{TP}}$, the more detailed structural changes described above also depart from the $\beta_{\mathrm{TP}} \mathrm{X}$-ray structure and approach the open $\beta_{\mathrm{E}} \mathrm{X}$-ray conformation, as can be seen by comparing the root mean square (r.m.s.) distances of the respective $\beta_{\mathrm{TP}}$ regions before and after $\gamma$-rotation to the $\beta_{\mathrm{E}} \mathrm{X}$-ray structure. For helix $\mathrm{H}$, a drop from $0.34 \mathrm{~nm}$ to $0.25 \mathrm{~nm}$ is observed; for the two strands of $\beta$-sheet 7 , the drop is from $0.15 \mathrm{~nm}$ to $0.13 \mathrm{~nm}$. The tilt angle between helices $\mathrm{B}$ and $\mathrm{C}$ increases from $25^{\circ}$ to $38^{\circ}$, approaching more than half way that of $\beta_{\mathrm{E}}\left(47^{\circ}\right)$.

Further conformational changes are expected from comparison of the $\beta_{\mathrm{TP}}$ subunit after forced rotation with the X-ray $\beta_{\mathrm{E}}$-structure; however, these changes are not (yet) seen in the simulations. Therefore, these motions (blue, Fig. 4) probably occur at slower time scales and/or are triggered by ATP release. They include further tilting of helix B, completion of the downward motion of helices 1 and 2, and significant motion of helix H. Closer analysis of the structural changes leads us to speculate that rotation of the lower half is completed only after ATP release, which should then trigger the motion of helix $\mathrm{H}$. This agrees with the finding that for $\beta_{\mathrm{E}}$, the expected reverse motion of helix $\mathrm{H}$ is not seen in the simulations and, hence, is also probably 


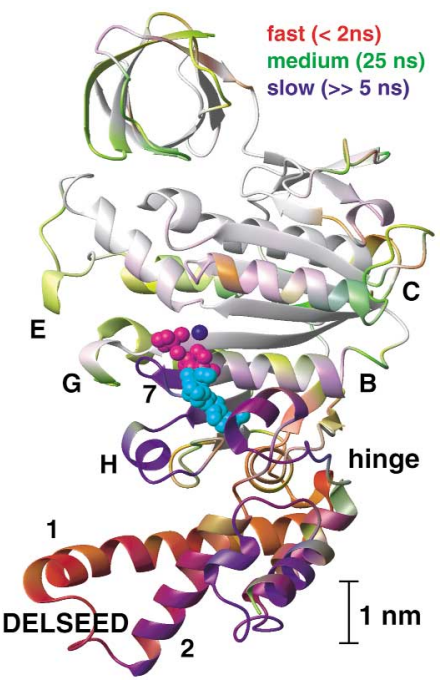

triggered by $\mathrm{ADP} /$ phosphate binding. Following the suggestion that the ATP concentration-independent delay of the $\gamma$-rotation after the recently observed $90^{\circ}$ substep of the hydrolysis cycle is defined by $\mathrm{ATP}+\mathrm{P}_{\mathrm{i}}$ release ${ }^{21}$, which, in turn, is tied to internal conformational motions, one may further speculate that these are among the slow motions not seen in our simulations.

Induced binding site changes reduce ATP binding affinity Focusing at the catalytic ATP-binding site (Fig. 5), a sequential retraction of residues $\beta \operatorname{Arg} 191, \alpha \operatorname{Arg} 373$ and $\beta \operatorname{Arg} 189$ from ATP is seen, which is quantified by the respective distance changes (blue, Fig. 5, inset). This sequence is initiated, immediately after $\gamma$-rotation, by a $0.5 \mathrm{~nm}$ shift of $\beta$ Arg 191 towards $\alpha_{\mathrm{TP}}$, which forms a new hydrogen bond to $\alpha$ Gln 163 , thereby weakening its interaction to ATP. Subsequently, initially strong hydrogen bonds (dashed lines, Fig. 5) between ATP and residues $\alpha$ Arg 373 and $\beta$ Arg 189 rupture, further decreasing ATP binding affinity. These two residues are essential for the promotion of rotational multisite catalysis ${ }^{26-28}$. We suggest $\beta$ Arg 191 as a further target for mutation studies.

Simultaneous to the first hydrogen bond rupture, the coordination of Thr 163 (green, Fig. 5) to the magnesium ion is lost. The whole process is compatible with the 'zipper-model'16, which postulates a sequential rupture of hydrogen bonds between ATP and the binding pocket. Assuming that changes of ATP binding affinity are dominated by the interaction between ATP and the binding pocket, a continuous ATP affinity decrease of $\beta_{\mathrm{TP}}$ can be inferred from the increasing interaction energy estimate between ATP and the binding pocket (black curve, Fig. 5, inset) as the net effect of the observed structural changes.

Also seen are changes at the $\alpha-\beta$ interfaces, which might contribute to the observed binding cooperativity ${ }^{8}$. In particular, with a delay of $2 \mathrm{~ns}$ after rotation of the $\gamma$-stalk, $\beta_{\mathrm{TP}} \operatorname{Arg} 260$,

Fig. 5 Changes at the $\beta_{\text {TP }}$ ATP binding site after the $120^{\circ}$ rotation and free dynamics. Shown are selected residues involved in ATP binding before (yellow, with ATP in light blue) and after enforced rotation and subsequent free dynamics (red, with ATP in blue). The backbones of part of the P-loop (Ala 158-Val 164) and of Glu 188-Glu 192 are colored green and orange, respectively. Residue numbers refer to $\beta_{\mathrm{TP}}$ if not annotated otherwise. The inset shows distances (blue) between the donor nitrogen atoms of three Arg residues involved in ATP binding and the respective ATP phosphates (reduced scale for Arg 191), as well as an estimate (black) for the change in interaction energy between ATP and the binding pocket in the course of the simulation. The period of enforced rotation is highlighted red.
Fig. 4 Sequence and timing of conformational changes in $\beta_{\text {TP. }}$ Shown are fast changes that mainly occur during forced rotation (red), slower ones that mainly occur during the subsequent free dynamics (yellow and green) and those not observed in the 7 ns simulation (blue) but are evident from the differences to the $\beta_{\mathrm{E}} \mathrm{X}$-ray structure. No significant changes are seen for the gray regions. The labels denote $\alpha$-helices, $\beta$-strands and regions referred to in the text.

which is important for intercatalytic-site cooperativity ${ }^{10}$, moves towards the position seen in $\beta_{\mathrm{E}}$, and a transient hydrogen bond is formed between $\beta_{\mathrm{TP}}$ Asp 386 and $\alpha_{\mathrm{DP}}$ Arg 398. Overall, $\gamma$-rotation is found to decrease the number of $\alpha_{\mathrm{DP}}-\beta_{\mathrm{DP}}$ and $\alpha_{\mathrm{DP}}-\beta_{\mathrm{TP}}$ van der Waals contacts.

\section{Conclusions and implications}

Our molecular dynamics simulations of forced $\gamma$-rotation suggest a sequence of structural changes that effect primary mechanochemical energy transfer steps within $\mathrm{F}_{1}$-ATP synthase. Many structural changes that are expected from the three X-ray intermediates are correctly reproduced. Because none of these motions have been artificially directed towards the expected target structure, the agreement shows that the necessarily accelerated rotation of the $\gamma$-stalk does not severely change their character.

In contrast to previous interpolations between the three known intermediates, the simulations provide a causal picture of a sequence of structural motions, with one triggering the next. Already within the simulation time span of $7 \mathrm{~ns}$, the structural changes proceed sufficiently far enough to transfer a significant amount of energy to the $\beta_{\mathrm{TP}}$-binding site, as estimated from its decreased ATP-binding affinity. This decrease is affected gradually by sequential and well-concerted retraction of three charged Arg residues from the bound ATP. Two main conformational energy transfer routes have been identified that couple $\gamma$-rotation to this retraction. Unexpectedly, a fast spontaneous closure of the empty $\beta$-subunit is seen during $\gamma$-rotation.

Some of the expected structural changes are not seen in the simulations and, therefore, probably occur at slower time scales than those accessible to our simulations. This finding allows classification of the structural changes into fast (nanoseconds) and slow changes. Necessarily, many questions remain open but can now be addressed in subsequent studies - for example, concerning the conformational changes triggered by ATP removal, ATP binding, ATP synthesis or phosphate binding, and concerning the specific role of the $\alpha$-subunits.

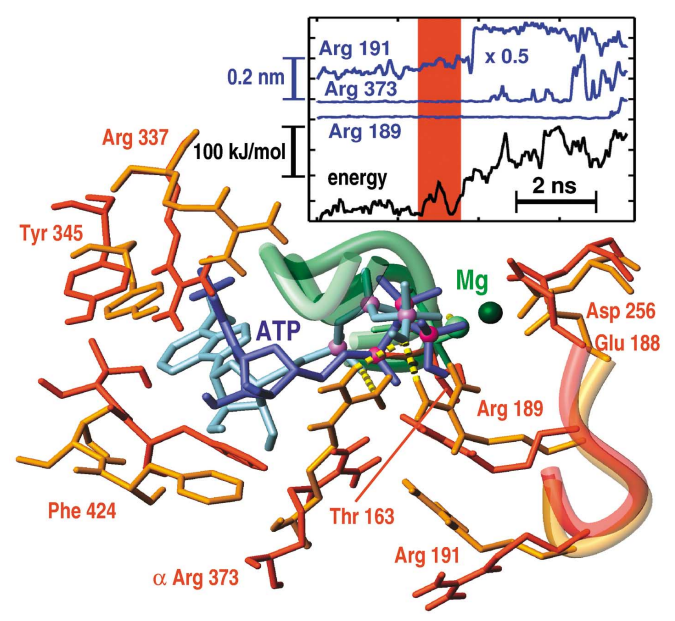




\begin{abstract}
Methods
Molecular dynamics simulations. All molecular dynamics simulations were carried out with the parallel MD program EGO29, the CHARMM force field ${ }^{30}$, a 1 fs integration step size and efficient computation of the full Coulomb interaction ${ }^{31}(\varepsilon=1)$. Nonpolar hydrogen atoms were described by compound atoms ${ }^{30}$, and the lengths of chemical bonds to hydrogen atoms were fixed. All surface water molecules were subjected to 'deformable boundary forces' 30,29 and coupled to a $300 \mathrm{~K}$ heat bath through stochastic forces (coupling constant $\beta=10 \mathrm{ps}^{-1}$ ). All other atoms were weakly coupled to a heat bath $\left(\beta=1 \mathrm{ps}^{-1}\right)$ via velocity rescaling. Mainly, 32 Power3 processors of an IBM RS/6000 SP3 parallel computer were used, totaling $\sim 280,000 \mathrm{~h}$ of cpu time.
\end{abstract}

System setup. The $(\alpha \beta)_{3} \gamma$ X-ray structure of $F_{1}$-ATPase from bovine heart mitochondria6 (Protein Data Bank entry 1BMF) was used. A structure with completely resolved $\gamma$-, $\delta$ - and $\varepsilon$-subunits is available ${ }^{7}$ but shows a shift of the $\gamma$-subunit relative to $(\alpha \beta)_{3}$. That shift is attributed to lattice contacts in the $F_{1}$ crystals ${ }^{7}$, presumably entailing distortions in the $\alpha_{T^{-}}, \beta_{\mathrm{E}^{-}}$and $\alpha_{\mathrm{E}^{-}}$-subunits and shifting the guanidinium group of $\alpha_{\text {TP }} \operatorname{Arg} 373$ in the active site ${ }^{7}$, which is important for cooperativity 26,27 . We do not expect the lack of the $\delta$ - and $\varepsilon$-subunits and of part of the $\gamma$-subunit to affect our results, because these parts do not contact the $(\alpha \beta)_{3}$-part (except for one hydrogen bond between $\gamma$ and $\beta_{D P}$ ). A third structure ${ }^{24}$ has recently become available; however, $\beta_{\mathrm{E}}$ is in an intermediate conformation and, therefore, not considered an appropriate start structure. The 1BMF structure was surrounded by 51,788 TIP3 water molecules ${ }^{32}$ and 297 ions $\left(\mathrm{Na}^{+}\right.$and $\left.\mathrm{Cl}^{-}\right)$at physiological concentration deterimined using SOLVATE ${ }^{29}$. For the placement of water molecules, energetic and steric criteria were used ${ }^{29}$. A nonspherical solvent volume with a minimum surface distance to the enzyme of $1.2 \mathrm{~nm}$ was chosen.

Equilibration. After 30,000 minimization steps, the system was equilibrated for $2.47 \mathrm{~ns}$, and the heavy atom r.m.s. deviations from the X-ray structure were monitored. The overall r.m.s. deviation showed the usual fast increase to $0.2 \mathrm{~nm}$ during the first $200 \mathrm{ps}$ and a slow drift for the subsequent $2 \mathrm{~ns}$; it stabilized at $0.27 \mathrm{~nm}$. The r.m.s. deviation of the ADP, ATP and empty binding sites were already stabilized after $1.0 \mathrm{~ns}$ at $0.2 \mathrm{~nm}, 0.13 \mathrm{~nm}$ and $0.22 \mathrm{~nm}$ respectively. The binding sites were defined as those residues which form hydrogen bonds or water bridges to the substrate, or are located close to the substrate - that is, $\alpha 343,344,349$ and $368-373$, and $\beta 158-164,188-192,256,257,260,337,345,416,418$, 421,424 and 425 . The only significant change seen is a move of ADP further into the binding pocket, such that the adjacent Phe 424 from $\beta_{D P}$ becomes more flexible. A missing ion not resolved in the X-ray structure probably causes this motion. The lower half of $\beta_{\mathrm{E}}$ tilted slightly towards the $\gamma$-stalk, forming a new hydrogen bond.

Force probe simulations. Torque was exerted onto the lower part of $\gamma$ (Fig. 1C). The $n$ backbone atoms of residues $16-41$ and $214-239$ with positions $x_{i}$ were subjected to a rotating potential, $\mathrm{V}=$ $1 / 2 \mathrm{k} \sum_{\mathrm{i}=1}^{n}\left[\Omega(\omega \mathrm{t}) \mathrm{x}_{\mathrm{i}}(\mathrm{t}=0)-\mathrm{x}_{\mathrm{i}}(\mathrm{t})\right]^{2}$. The rotation matrix $\Omega$ moved the minima of $\mathrm{V}$ along concentric circles around the symmetry axis of the complex. A stiff spring coefficient of $k=7 \mathrm{~N} \mathrm{~m}^{-1}$ has been used. The torques $\Theta_{i}(t)$ exerted on the individual atoms were computed from the deflection of the actual atomic positions $x_{i}(t)$ from the respective minima, $\Theta_{i}(t)=\Omega(\omega t) x_{i}(t=0) \times k\left[\Omega(\omega t) x_{i}(t=0)-x_{i}(t)\right]$ Torque profiles per residue were obtained from the summed atomic torques, averaged over 50 ps intervals.
Post-simulation analysis. The change of interaction energy between the $\beta_{\text {TP }}$ binding pocket and ATP was estimated from the sum of the van der Waals and the Coulomb interactions between ATP and all binding pocket atoms within a $0.8 \mathrm{~nm}$ vicinity. Focusing at timing rather than accurate energies, $\varepsilon=5$ was used as a rough description of dielectric screening. The timing of the motion of each $\beta_{\mathrm{TP}}$-residue was characterized by its relative average location $\left\langle\left(x_{e}-x_{s}\right)\left(x(t)-x_{s}\right) /\left|x_{e}-x_{s}\right|^{2}\right\rangle_{t}$ in configurational space along the distance vector connecting the equilibrated start configuration $x_{s}(t=2.5 \mathrm{~ns})$ and a final configuration $x_{e}(t=6.78 \mathrm{~ns})$. The size of structural motions was quantified via r.m.s. distances between the structure averaged over the last nanosecond of the simulation and the X-ray structure. Figures were created with $\mathrm{MOLMOL}^{33}$ (Figs. 1a, 2-4) and RASMOL ${ }^{34}$ (Fig. 1b).

\section{Acknowledgments}

We thank W. Junge, A. Engel, B. de Groot, B. Heymann, K. Schulten, W. Allison, D. Chandler, V. Helms, M. Hofmann, K. Kinosita, V. Knecht, R. Lang, G. Oster, G. Schröder and H. Wang for stimulating discussions and for critical reading of the manuscript; B. de Groot for help with the GROMACS program package; and G. Schneider and $\mathrm{O}$. Haan for their support. Computer time was provided by the Göttingen computer center (GWDG) and the Paderborn center for parallel computing $\left(\mathrm{PC}^{2}\right)$.

\section{Competing interests statement}

The authors declare that they have no competing financial interests.

Correspondence should be addressed to H.G. email: hgrubmu@gwdg.de

Received 1 November, 2001; accepted 21 December, 2001.

1. Mitchell, P. Nature 191, 144-148 (1961).

2. Boyer, P.D. Biochim. Biophys. Acta 1140, 215-250 (1993).

3. Duncan, T.M., Bulygin, V.V., Zhou, Y., Hutcheon, M.L. \& Cross, R.L. Proc. Natl. Acad. Sci. USA 92, 10964-10968 (1995).

4. Sabbert, D., Engelbrecht, S. \& Junge, W. Nature 381, 623-625 (1996).

5. Noji, H., Yasuda, R., Yoshida, M. \& Kinosita, K. Nature 386, 299-302 (1997)

6. Abrahams, J.P., Leslie, A.G.W., Lutter, L. \& Walker, J.E. Nature 370, 621-628 (1994).

7. Gibbons, C., Montgomery, M.G., Leslie, A.G.W. \& Walker, J.E. Nature Struct. Biol. 7, 1055-1061 (2000).

8. Weber, J. \& Senior, A.E. Biochim. Biophys. Acta 1319, 19-58 (1997)

9. Pänke, O. \& Rumberg, B. Biochim. Biophys. Acta 1322, 183-194 (1997).

10. Ren, G. \& Allison, W.S. Biochim. Biophys. Acta 1458, 221-233 (2000).

11. Hara, K.Y. et al. J. Biol. Chem. 275, 14260-14263 (2000).

12. Boyer P. Annu. Rev. Biochem. 66, 717-749 (1997).

13. Engelbrecht, S. \& Junge, W. FEBS Lett. 414, 485-491 (1997)

14. Cherepanov, D.A., Mulkidjanian, A.Y. \& Junge, W. FEBS Lett. 449, 1-6 (1999).

15. Weber, J., Nadanaciva, S. \& Senior, A.E. FEBS Lett. 483, 1-5 (2000).

16. Oster, G. \& Wang, H.Y. Biochim. Biophys. Acta 1458, 482-510 (2000)

17. Grubmüller, H., Heymann, B. \& Tavan, P. Science 271, 997-999 (1996)

18. Izrailev, S., Stepaniants, S., Balsera, M., Oono, Y. \& Schulten, K. Biophys. J. 72, 1568-1581 (1997).

19. Dunn, S.D. \& Futai, M. J. Biol. Chem. 255, 113-118 (1980).

20. Miwa, K. \& Yoshida, M. Proc. Natl. Acad. Sci. USA 86, 6484-6487 (1989).

21. Yasuda, R., Noji, H., Yoshida, M., Kinosita, K. \& Itoh, H. Nature 410, 898-904 (2001)

22. Pänke, O., Cherepanov, D.A., Gumbiowski, K., Engelbrecht, S. \& Junge, W. Biophys. J. 81, 1220-1233 (2001)

23. Weber, J., Hammond, S.T., Wilke-Mounts, S. \& Senior, A.E. Biochemistry 37 608-614 (1998)

24. Menz, R.I., Walker, J.E. \& Leslie, A.G.W. Cell 106, 331 (2001)

25. Heymann, B. \& Grubmüller, H. Chem. Phys. Lett. 303, 1-9 (1999).

26. Nadanaciva, S., Weber, J., Wilke-Mounts, S. \& Senior, A.E. Biochemistry 38 15493-15499 (1999)

27. Le, N.P. et al. Biochemistry 39, 2778-2783 (2000)

28. Nadanaciva, S., Weber, J. \& Senior, A.E. Biochemistry 38, 7670-7677 (1999).

29. Eichinger, M., Heller, H. \& Grubmüller, H. In Workshop on molecular dynamics on parallel computers. (eds Esser, R. et al.) 154-174 (World Scientific, Singapore; 2000). 30. Brooks, B.R. et al. J. Comp. Chem. 4, 187-217 (1983).

31. Eichinger, M., Grubmüller, H., Heller, H. \& Tavan, P. J. Comp. Chem. 18, 1729-1749 (1997)

32. Jorgensen, W.L., Chandrasekhar, J. \& Madura, J.D. J. Chem. Phys. 79, 926-935 (1983).

33. Koradi, R., Billeter, M., \& Wüthrich, K. J. Mol. Graphics 14, 51-55 (1996).

34. Sayle, R.A. \& Milnerwhite, E.J. Trends Biochem. Sci. 20 374-376 (1995). 